\title{
Placental histopathology in high-risk pregnancy
}

\section{Original Research Article}

\author{
Kumar S. ${ }^{1}$, Sudarshan V. ${ }^{2}$ \\ ${ }^{1}$ Dr. Sunil Kumar, Senior Resident, Department of Pediatrics, Kalpana Chawla Government Medical College, Karnal, \\ Haryana, ${ }^{2}$ Dr. Vijaya Sudarshan, Retd. Professor and Head, Department of Pathology, Pt JNM Medical College, Raipur \\ (C.G), India.
}

Corresponding Author: Dr. Sunil Kumar, Senior Resident, Department of Pediatrics, Kalpana Chawla Government Medical College, Karnal, Haryana. Email: drsunilx@yahoo.com

\begin{abstract}
Introduction: Placenta is a vital organ for the wellbeing of the fetus. Pregnancies with preganancy induced hypertension $(\mathrm{PIH})$, diabetes, anemia etc. are associated with adverse neonatal outcome. Objective: This study explores the histopathological changes in the placenta of the mothers with conditions known to have an adverse effect on fetal well-being. Methods: The study was conducted at the Department of Obstetrics and Gynecology and Department of Pediatrics, Raipur. In the study, 96 placenta (with their membrane and cords) were included. Among 96 placenta, 72 were cases with maternal risk factors viz. bad obstetric history, maternal anemia, PIH, diabetes, history of handling, advanced age and 24 placenta were controls without significant risk factors and were studied for histopathological changes. Results: Women with a history of fetal-neonatal loss in the past showed more of chorioamnionitis (33\%) in their placenta. Placental pathology of 20 anemic women was compared with non-anemic controls. Anemic mothers found to have more of infarction (60\%), calcification (30\%) and villous fibrosis (100\%). Calcification was found in 33\% of cases. Syncytial knots (p-0.001) and Cytotrophoblast proliferation $(\mathrm{p}<0.01)$ were statistically significant with females with PIH as compared to controls and the incidence of villous fibrosis with diabetic mother was statistically significant (p-0.04). Conclusion: In the present study, it was found that the placenta in anemic mothers showed evidence of uteroplacental ischemia and early senescence. Diabetic mothers showed signs of dysmaturity and increased fibrinoid necrosis with evidence of placental insufficiency responsible for increased morbidity in the newborn of diabetic mothers.
\end{abstract}

Keywords: Placenta, High-risk Pregnancy, Pathology.

\section{Introduction}

The placenta is responsible for gaseous and nutrition transfer from mother to the fetus as well as disposable of waste products of the fetus. It is a metabolic and endocrine organ of supreme importance for establishment and maintenance of the pregnancy. Since it is the only point of contact between maternal and fetal tissues anything which affects the fetus can do so only through the placenta. It is also important for immunological acceptance of the fetus by the mother [1].

Origin of the term placenta is controversial. Some give this credit to Gabriele de Falloppio (1532-1562), after whom fallopian tubes are named. While some believe that the term "Placenta" was introduced by Realdus Columbus (1559) and was derived from the Latin word "Palkus" meaning circular/flat cake. "Secunidinae" was another popular name for it in early days [1]. Any maternal diseases, acute or chronic, have its effect on fetus by

Manuscript received: $10^{\text {th }}$ June 2018

Reviewed: $20^{\text {th }}$ June 2018

Author Corrected: $27^{\text {th }}$ June 2018

Accepted for Publication: $30^{\text {th }}$ June 2018

Obsgyne Review: Journal of Obstetrics and Gynecology altering the placental metabolism and transfer mechanism. Any such compromise is likely to leave its impression on the placenta, which can be detected by pathological and ultrasonography examinations. But one should not be over expecting from placental examinations since placental change areamanifestation of primary compromise rather than the primary cause of neonatal morbidity [2].

Various studies done in past found that placental pathological changes associated with maternal risk factors like hypertension $[3,4]$, diabetes[5, 6], anemia [7,8,9] and bad Obstetric History $[10,11,12]$ subsequently affect the neonatal outcome. Since, the placenta is the connecting link between the mother and the fetus, any pathophysiological process or maternal condition which has a negative impact on the baby is likely to leave its mark on the placenta. Hence, this study was designed to explore the histopathological changes in the placenta of the mothers with conditions known to have an adverse effect on fetal well-being.

Available online at: www.medresearch.in 39 | P a g e 


\section{Material and Methods}

A study aimed at finding the association between the histopathological changes in placenta and outcome in the neonate was conducted at the Department of Obstetrics and Gynecology (with newborns admitted at Department of Pediatrics) and histopathological examination was done in the Department of Pathology of a Medical College of Raipur.

Ethical approval: The study protocol was reviewed by the Ethical committee of the Institutional Review Board and was granted ethical clearance. Written informed consent was taken from the subject or their family members.

Study subjects: In the present study, 96 placenta (with their membrane and cords) were included. Among 96 placenta, 72 were cases with maternal risk factors viz. bad obstetric history (12 cases), maternal anemia (20 cases), pregnancy-induced hypertension (PIH) (18 cases), diabetes (6 cases), history of handling (10), advanced age (6 cases) and 24 placenta were taken as controls without significant risk factors. These factors were studied for histopathological changes.

Inclusion criteria: The following inclusion criteria were used-

PIH: In this group, those patients were included, who had a blood pressure recording of $140 / 90 \mathrm{~mm}$ of $\mathrm{Hg}$ or more with or without edema and/or albuminuria.

Pregnancy with anemia: In this group, the patient who had hemoglobin level $<8 \mathrm{gm} \%$ were included.

Pregnancy with diabetes: This group included patients with fasting blood glucose $>90 \mathrm{mg} / 100 \mathrm{mt}$ and postprandial blood glucose (after 2 hours of ingestion of $100 \mathrm{gm}$ of glucose) $>120 \mathrm{mg} / 100 \mathrm{ml}$.

Bad Obstetric History: Mothers with a history of previous fetal or neonatal loss.

\section{Original Research Article}

Sampling technique: Convenient sampling was used. Females fulfilling the inclusion criteria were included in the study.

Data Collection: Name of the patient, age address, detailed medical, obstetrics and menstrual history were obtained. General examination (including pallor, BP) and systemic examination was done. Any systemic disease, if present was noted.

Examination of the placenta: Placenta were collected from mothers delivering at the Hospital. After collecting, the placenta was examined for gross findings such as infraction or calcification. Then they were fixed in $10 \%$ formalin for 48 hours.

After 48 hours of fixation in formalin, $5 \mathrm{~cm}$ of tissue strips were taken from the following areas: umbilical cord, central portion of the placenta, peripheral portion of the placenta, any gross lesion or representative area, extraplacental membranes. Sections of the tissues were taken for routine paraffin embedding.

After embedding in paraffin wax sections of 5-7 micrometer were taken with the microtome. Sections were stained with Hematoxylin and Eosin stain. Placenta, cord and membrane were examined for the following pathological changes: chorioamnionitis, infarction, villitis, funisitis, syncytial knots, fibrinoid necrosis, cytotrophoblast proliferation, villous stromal fibrosis, calcification and umbilical thrombosis

Statistical analysis: Data thus collected, was analyzed using chi-square test ( $\chi^{2}$ test) for the significance of the difference in the proportion of pathological change in various groups studied.

The test was applied and $\mathrm{p}$ value was calculated using MS Excel 2003, Data analysis tool. p value $<0.05$ was considered as statistically significant.

\section{Results}

Women with history of fetal or neonatal loss in the past showed more of infarction ( $66 \% \mathrm{vs} .46 \%)$, chorioamnionitis (33\% vs. $15 \%)$, and villitis (33\% vs. $13 \%)$ in their placentas while incidence of calcification was found to be reduced (16.7\% vs $37.5 \%)$.

Cytotrophoblast proliferation was statistically higher in women with a history of fetal or neonatal loss in the past (Table 1)

Placental pathology of 20 anemic women was compared with non-anemic controls. Anemic mothers found to have more of infarction (60\% vs. $45 \%)$, Syncytial knotting (40\% vs. 16\%), villous stromal fibrosis (90\% vs. $66 \%)$ and calcification (30\% vs. $37 \%$ ). Fibrinoid necrosis was found in $60 \%$ cases while $20 \%$ had cytotrophoblastic proliferation. 
Original Research Article

Table-1: Comparison of placental pathology in mother with bad obstetric history as compared to healthy mother

\begin{tabular}{|c|c|c|c|c|c|}
\hline Pathology & \multicolumn{2}{|c|}{ Bad Obstetric History } & \multicolumn{2}{c|}{ Healthy mother } & p value \\
\hline Chorioamnionitis & 4 & $33.3 \%$ & 4 & $16.7 \%$ & 0.25 \\
\hline Infarction & 8 & $66.7 \%$ & 11 & $45.8 \%$ & 0.23 \\
\hline Villitis & 4 & $33.3 \%$ & 2 & $8.3 \%$ & 0.05 \\
\hline Funisitis & 0 & $0.0 \%$ & 2 & $8.3 \%$ & 0.30 \\
\hline Syncytial knots & 4 & $33.3 \%$ & 4 & $16.7 \%$ & 0.25 \\
\hline Fibrinoid Necrosis & 8 & $66.7 \%$ & 16 & $66.7 \%$ & 0.90 \\
\hline Cytotrophoblast proliferation & 2 & $16.7 \%$ & 0 & $0.0 \%$ & $* 0.04$ \\
\hline Villous strom Fibrosis & 10 & $83.3 \%$ & 16 & $66.7 \%$ & 0.29 \\
\hline Calcification & 2 & $16.7 \%$ & 9 & $37.5 \%$ & 0.20 \\
\hline Umbilical thrombosis & 0 & $0.0 \%$ & 2 & $8.3 \%$ & 0.30 \\
\hline
\end{tabular}

* p value $<0.05$ : statistically significant

The results of the current study suggest that increased chorioamnionitis is found in diabetic mothers' placentas, although not significant statistically. Increased incidence of Fibrinoid necrosis (100\%) syncytial knotting (66\%), cytotrophoblastic proliferation (50\%), and villous fibrosis $(100 \%)$ was found. Calcification was found in 33\% of cases.

In the present study, cases with $\mathrm{PIH}$ had more of infarction (55\% vs. 45\%), fibrinoid necrosis (72.2\% vs.66.7\%), cytotrophoblastic proliferation $(50 \%$ vs. $0 \%)$, villitis $(33.3 \%$ vs. $8 \%)$ and villous stromal fibrosis $(83 \%$ vs. $66 \%)$ in their placenta. Calcification was present in 50\%. Syncytial knots (p-0.001) and Cytotrophoblast proliferation $(\mathrm{p}<0.01)$ were statistically significant with females with PIH as compared to controls and the incidence of villous fibrosis with diabetic mother was statistically significant (p-0.04) (Figure 1).

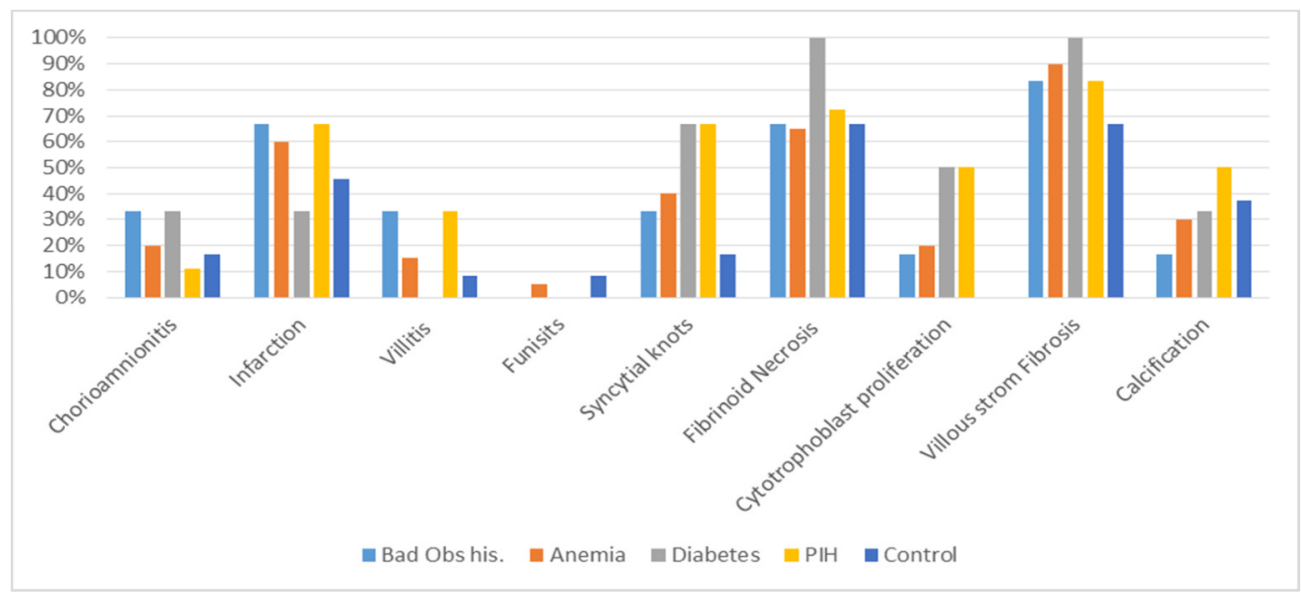

Figure-1: Comparison of placental pathology in mother with bad obstetric history, diabetes, anemia, PIH and control (healthy)

\section{Discussion}

Human placenta is hemochorial organ formed by decidua basalis of mother and chorion frondosum of the fetus. The placenta develops as a specialized region of the complete sac-like structure surrounding and enclosing the baby - the chorion - together with adjacent endometrial tissues. In the present study, it was found that women with a history of fetal or neonatal loss in the past showed more of chorioamnionitis, infarction, and villitis in their placentas while the incidence of calcification was found to be reduced. One of the studies done by Edwin R. Guzman et al[11] found an association of chorioamnionitis with recurrent pregnancy. Increased incidence of chorioamnionitis indicates an association of placental infection with recurrent reproductive loss and the possibility of recurrence of the lesion. Also, association with other factors which are associated with recurrent 


\section{Original Research Article}

pregnancy loss like cervical shortening may be another reason for this association. These factors are also associated with chronic inflammatory changes like villitis and vascular lesions like infarction which is a reasonable explanation of the higher incidence of villitis and infarction found in the study [11,12]. Higher incidence of infarction indicates the role of uteroplacental ischemia while an increase in villitis role of chronic immune reaction in the recurrent reproductive loss.Recurrent nature of the inflammatory lesions of the placenta has been confirmed by the study of Ghdini A[12] which is found in mothers with recurrent pregnancy loss in the current study.

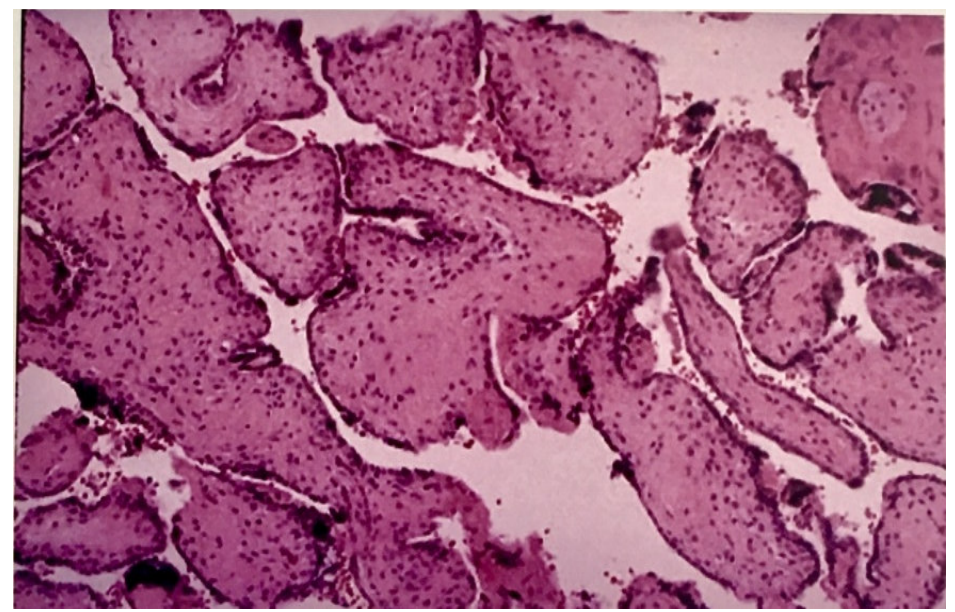

Figure 1: Photomicrograph of placenta showing villous fibrosis.

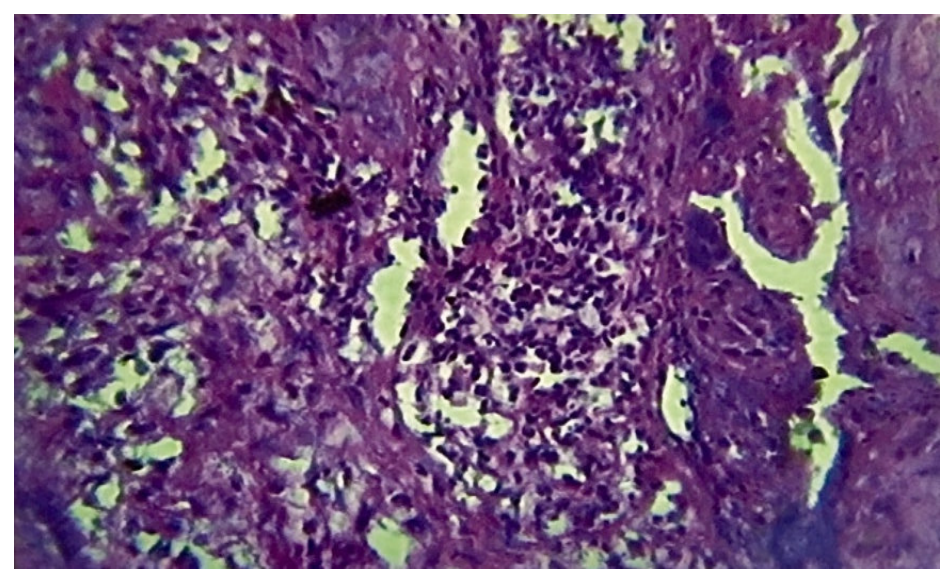

Figure 2 : photomicrograph of placenta showing villitis.

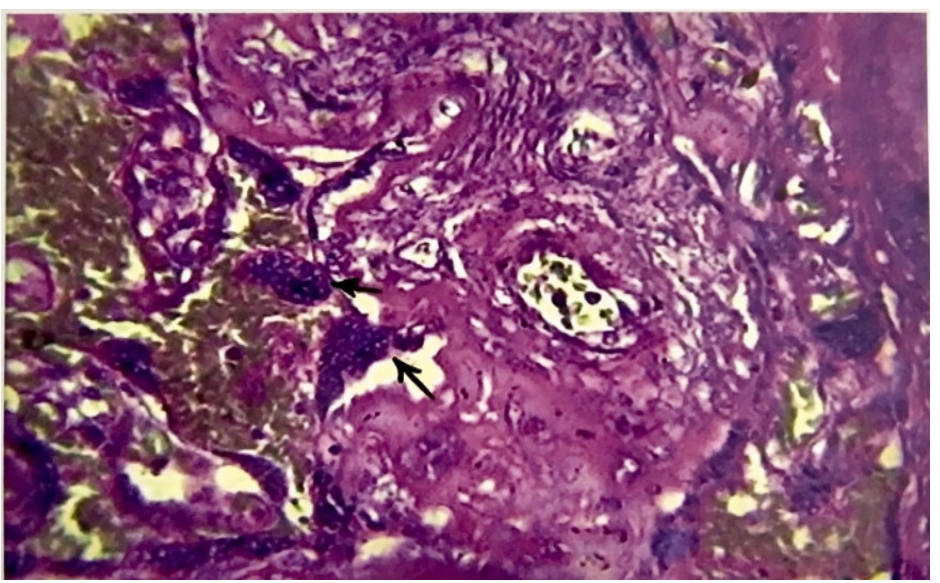

Figure 3: Photomicropgraph of placenta showing syncytial knotting. 


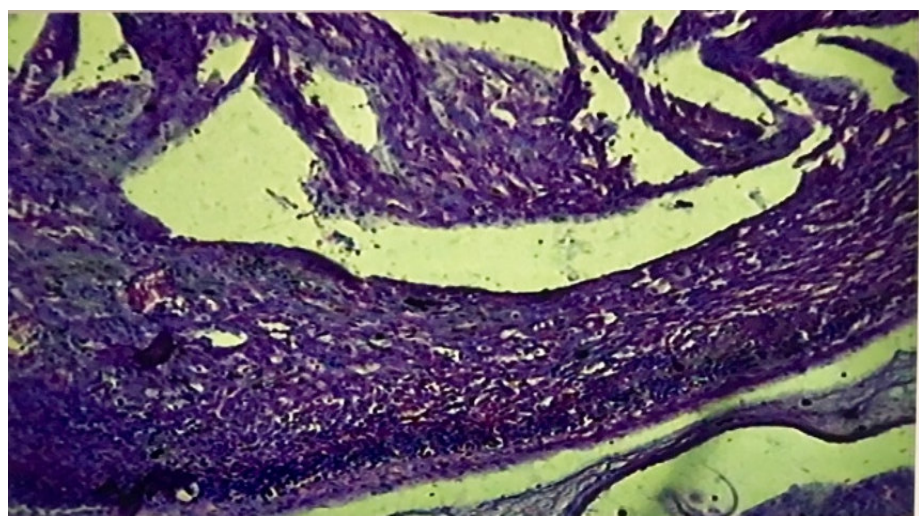

Figure 4: Photomicropgraph of placenta showing chorioamnionitis.

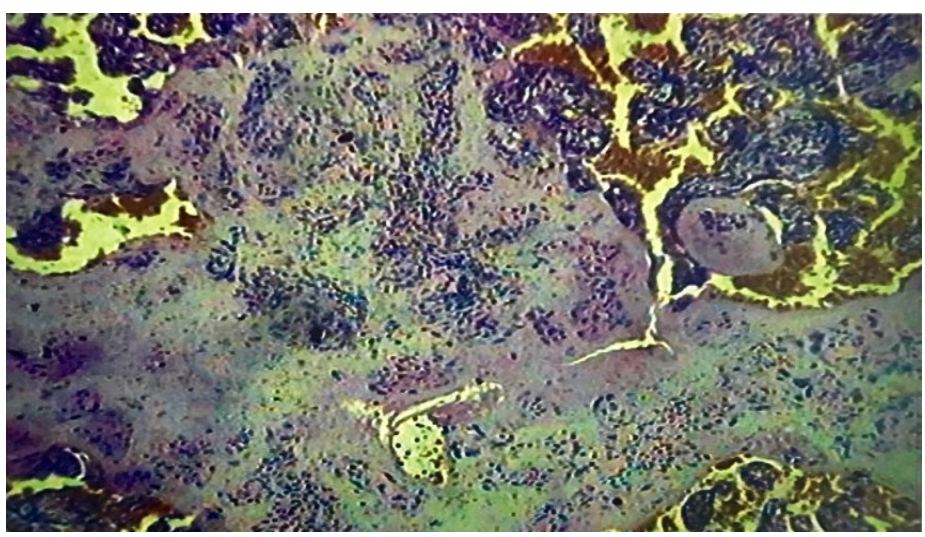

Figure 5: Photomicropgraph of placenta showing infarction.

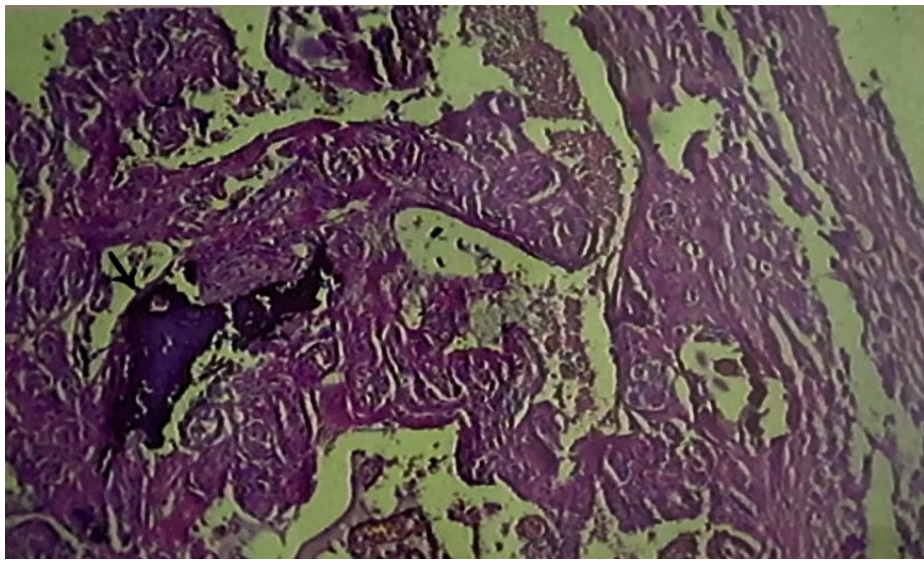

Figure 6: Photomicropgraph of placenta showing calcification.

In the present study, anemic mothers found to have more of infarction, Syncytial knotting, villous stromal fibrosis and calcification. Rangnekar et al [7] in a study of placental changes in pregnancy anaemia found that, increased villous vascularity and decreased incidence of excess syncytial knot formation, suggest a possibility of an adequate compensatory capacity of patients. There was increased villous stromal fibrosis, endarteritis obliterans and decreased fibrinoid necrosis and intervillous calcification when compared to controls. Rusia, U et al (1998) [8] found increased fibrinoid necrosis, basement membrane thickening villous fibrosis and calcification, in anemic placentas. The present study has more or less similar findings increased incidence of infarctions can be explained uteroplacental ischemia induced by anemia. Fibrinoid necrosis, villous fibrosis is thought to reflect early senescence. This study finds increased chorioamnionitis although not significant statistically. 


\section{Original Research Article}

Increased incidence of Fibrinoid necrosis, syncytial knotting, Cytotrophoblastic proliferation and villous fibrosis was found in diabetics women in the present study. Anjali R Madoskar et al [13] found increased syncytial knotting in $80 \%$, fibrinoid necrosis in $100 \%$, increased basement membrane thickness in $100 \%$, cytotrophoblastic hyperplasia in $60 \%$, villous fibrosis in $100 \%$ diabetes cases while none in controls. Fibrinoid necrosis was found to be increased in diabetic placentas by Evers IM et al [5] also. Increased chorioamnionitis found in the study is most probably an independent finding since there is no correlating pathophysiological mechanism. While increased cytotrophoblast proliferation indicates placental dysmaturity which is highly typical in placentas of diabetic mothers. No increase in calcification was found as in the study conducted by Fox in [14].

In the present study, cases with PIH had more of infarction, fibrinoid necrosis, cytotrophoblastic proliferation, villitis and villous stromal fibrosis in their placenta. Calcification was present in 50\%. Anjali Satosaret al [15] found increased fibrinoid necrosis in 50\%, cytotrophoblastic hyperplasia in 52\%, villous fibrosis in 53\%, infarction in (58\%) cases of PlH. Rihard L Naeye[16] found an increased incidence of infarction, fibrinoid necrosis in placenta with PIH. He also found that infarction is associated with increased risk of newborn mortality. Fox H., [14] found infarction in 33-60\% of placentas depending on the severity of hypertension. Manifestations of reduced maternal blood flow in the form of infarction, cytotrophoblast hyperplasia and reduced fetal blood flow in the form of and syncytial knotting and villous fibrosis have been found in the previous studies. This was also proved by Doppler studies showing reduced fetal and maternal blood flow in the placenta in cases of $\mathrm{PIH}[17]$.

\section{Conclusion}

In the present study, it was found that the placenta in anemic mothers showed evidence of uteroplacental ischemia and early senescence. Diabetic mothers showed signs of dysmaturity and increased fibrinoid necrosis with evidence of placental insufficiency responsible for increased morbidity in the newborn of diabetic mothers.

Placenta in mothers with a history of PIH showed a pattern consistent with reduced maternal blood flow and uteroplacental ischemia, with changes in the villous portion of the placenta secondary to a reduction in the maternal blood flow as a compensatory response.

Contributotors: SK was involved in sample collection, review of literature, data analysis, drafting of articles.

VS: Pathlogical examination, Study design, manuscript review.

What this study adds:Various placental abnormalities are associated with increased neonatal morbidity.

Recommendation: More robust studies should be conducted to esplore the clinicopathological correlation between high risk pregancny and placental histopatholgy.

Funding: Nil, Conflict of interest: Nil Permission from IRB: Yes

\section{References}

1. Norwitz ER, Schust DJ, Fisher SJ. Implantation and the survival of early pregnancy. DOI:10.1056/NEJMra000763

2. Fox H. The histopathology of placental insufficiency. J Clin Pathol Suppl (R Coll Pathol). 1976;10:1-8.
3. Redline RW, Patterson P. Patterns of placental injury. Correlations with gestational age, placental weight, and clinical diagnoses. Arch Pathol Lab Med. 1994 Jul;118 (7): 698-701.

4. Nahar L, Nahar K, Hossain MI, et al. Placental changes in pregnancy induced hypertension. Mymensingh Med J. 2013 Oct;22(4):684-93.

5. Evers IM, Nikkels PG, Sikkema JM, Visser GH. Placental pathology in women with type 1 diabetes and in a control group with normal and large-for-gestational-age infants. Placenta. 2003 Sep-Oct;24(8-9):819-25.

6. Saha S, Biswas S, Mitra D, Adhikari A, Saha C. Histologic and morphometric study of human placenta in gestational diabetes mellitus. Ital J AnatEmbryol. 2014; 119 (1):1-9.

7. Rangnekar AG, Darbari R. Placental changes in pregnancy anaemia : A study of one hundred cases: J Obstet Gynaecol India.1993; 43(4): 473-8.

8. Rusia U, Bhatia A, Kapoor S, et al. Placental morphology \& histochemistry in iron deficiency anemia. Indian J Med Res. 1988 May;87:468-74.

9. Singla PN, Chand S, Khanna S, Agarwal KN. Effect of maternal anaemia on the placenta and the newborn infant. Acta Paediatr Scand. 1978 Sep;67(5):645-8.

10. Boyd TK, Redline RW. Chronic histiocytic intervillositis: a placental lesion associated with recurrent reproductive loss. Hum Pathol. 2000 Nov;31(11):1389-96. 
11. Guzman ER, Shen-Schwarz S, Benito C, et al. The relationship between placental histology and cervical ultrasonography in women at risk for pregnancy loss and spontaneous preterm birth. Am J Obstet Gynecol. 1999 Oct; 181(4):793-7.

12. Ghidini A, Salafia CM. Histologic placental lesions in women with recurrent preterm delivery. OI:10.1111/j. 0001-6349.2005.00694.x

13. Anjali R. Masodkar et al. Histopathology ofplacenta and its correlation worth outcome: Journal of Obstetrics and Gynaecology of India, 1985: 35(2): 294-300.

14. Fox H. Pathology of the placenta in maternal diabetes mellitus. Obstet Gynecol. 1969 Dec;34(6):792-8.

\section{Original Research Article}

15. Anjali Satosar. Histologic correlates of viral and bacterial infection of the placenta associated with severe morbidity and mortality in new born. Human Pathology 2006; 35(5): 536-545. Doi: 10.1016/J. Humpath. 2004. 01.015 .

16. Naeye RL, Friedman EA. Causes of perinatal death associated with gestational hypertension. Am J Ob Gyn. 1979;133(8):8-10. Doi: 10.1016/0002-9378(79)90402-2.

17. Arias F, Rodriquez L, Rayne SC, Kraus FT. Maternal placental vasculopathy and infection: two distinct subgroups among patients with preterm labor and preterm ruptured membranes. Am J Obstet Gynecol. 1993 Feb;168 (2):585-91.

\section{How to cite this article?}

Kumar S, Sudarshan V. Placental histopathology in high-risk pregnancy.Obs Rev:J obstet Gynecol 2018;4(2):39-45.doi: 10.17511/joog.2018.i02.04. 